\title{
La eficacia del etanol intraglandular en hiperparatiroidismo secundario se limita a una sola glándula hiperplásica
}

\author{
Effectiveness of intraglandular ethanol in \\ secondary hyperparathyroidism limited to one only \\ hyperplastic gland
}

\author{
César A. Restrepo, Campo Elías Castillo, María Fernanda Sanz, \\ Consuelo Vélez • Manizales, Caldas (Colombia)
}

\section{Resumen}

Objetivo: determinar la eficacia del etanol al 99,5\% en reducción de los niveles de PTH intacta (PTHi) al aplicarlo directamente en las glándulas paratiroides hiperplásicas de sujetos con enfermedad renal crónica (ERC) estadio 5 en terapia dialítica con hiperparatiroidismo secundario

Diseño: estudio de intervención sin grupo control.

Analisis estadístico: se utilizaron pruebas de tendencia central y de dispersión (media, desviación estándar) y se realizó la comparación de medias a través de la prueba $t$ de Student.

Pacientes, material y métodos: durante un periodo de recolección de dos años se identificaron sujetos con ERC estadio 5 en terapia dialítica (hemodiálisis o diálisis peritoneal), mayores de 18 años, quienes presentaran niveles de PTHi séricos mayores a $600 \mathrm{pg} / \mathrm{mL}$, e hiperplasia de glándulas paratiroides autónoma (volumen mayor a $500 \mathrm{~mm}^{3}$ ) confirmada por ultrasonografía de cuello. Bajo visión ecográfica se les aplicó etanol en cada glándula hiperplásica, volumen dependiente de tamaño glandular para un máximo de dos aplicaciones por glándula con un intervalo de 30 días; si habían más de dos glándulas alteradas sólo se permitió la infiltración de dos glándulas por sesión. Quince sujetos fueron sometidos a la terapia, en el grupo 1 (G1) se incluyeron cinco sujetos con una sola glándula hiperplásica, el grupo 5 (G5) incluyó diez sujetos con más de una glándula hiperplásica: grupos 2, 3 y 4 cada uno con tres, dos y cinco sujetos con dos, tres y cuatro glándulas hiperplásicas respectivamente. Los niveles de PTHi se evaluaron a los 30, 60, 90 y 120 días posterior a la última aplicación, al igual que las variables calcio, fósforo, producto calcio-fósforo y hemoglobina. La fosfatasa alcalina se evaluó inicialmente y a los tres meses.

Resultados: la administración de etanol intraglandular disminuyó los valores de PTHi significativamente en la población total de sujetos desde un valor basal de $1263 \pm 554 \mathrm{pg} / \mathrm{mL}$ a $1065 \pm 437 \mathrm{pg} / \mathrm{mL}$ (valor p: 0.08 ), $989 \pm 568 \mathrm{pg} / \mathrm{mL}$ (valor p: 0.02 ), $1028 \pm 643 \mathrm{pg} / \mathrm{mL}$ (valor p: 0.04 ) y $1139 \pm 749 \mathrm{pg} / \mathrm{mL}$ (valor p: 0.194) a los intervalos previamente descritos. En el análisis de grupos se observó que sólo el G1 presentó una reducción significativa en los valores de PTHi, con disminución de un valor basal promedio de $910 \pm 508 \mathrm{pg} / \mathrm{mL}$ a $693 \pm 432$ (valor p: 0.04 ), $534 \pm 426$ (p: 0.006 ), $330 \pm 379$ (p: 0.01 ) y $322 \pm 75$ (p: 0.44). En los otros grupos consolidados en el grupo 5 (G5) con más de una glándula hiperplásica los valores de PTHi no se modificaron en forma importante, siendo su valor inicial 1430 $\pm 520 \mathrm{pg} / \mathrm{mL}$ y sus valores posteriores $1251 \pm 313$ (p: 0.287$), 1241 \pm 482(\mathrm{p}: 0.255), 1378 \pm 416(\mathrm{p}$ : 0.635 ) y $1489 \pm 591$ (p: 0.869). Los valores de calcio, fósforo, producto calcio-fósforo y fosfatasa alcalina, se disminuyeron en todos los grupos en forma no significativa, probablemente por el corto tiempo de seguimiento. El evento adverso más frecuente fue dolor y sensación de quemazón que se extendió de 5-10 segundos en el área inyectada, con presencia de hipotensión en un sujeto y disfonía en tres sujetos, resolviéndose con fonoaudiología en el curso de tres meses. Todos los sujetos con falla terapéutica fueron referidos a cirugía de cabeza y cuello con intención de paratiroidectomía, con hallazgos de grandes bandas fibrosas durante la cirugía en los ocho pacientes operados a la fecha.
Dr. César A. Restrepo Valencia: Internista Nefrólogo, Profesor Asociado Universidad de Caldas; Dr. Campo Elías Castillo Pinilla: Radiólogo, Profesor Universidad de Caldas Licenciada María Fernanda Sanz Scovino: Enfermera Jefe, Certificada en Nefrología; Licenciada Consuelo Vélez Álvarez: Enfermera, Epidemióloga, PhD en Salud Pública. Docente Asociada Universidad de Caldas. Manizales, Caldas (Colombia).

Correspondencia. Dr. César A. Restrepo V. E-mail: caugustorv@une.net.co Recibido: 31/VII/2012 Aceptado: 7/III/2013 
Conclusión: el etanol intraglandular controla eficazmente el hiperparatiroidismo secundario en sujetos con alteración de una glándula hiperplásica. Sujetos con más de una glándula hiperplásica deben ser manejados quirúrgicamente o con un calcimimético oral tipo Cinacalcet. (Acta Med Colomb 2013; 38: 61-67)

Palabras clave: hiperparatiroidismo secundario, enfermedad renal crónica, etanol.

\section{Abstract}

Objective: determine the efficacy of ethanol 99.5\% in intact PTH (PTHi) levels reduction when applied directly in the hyperplastic parathyroid glands of subjects with chronic kidney disease (CKD) stage 5 on dialysis with secondary hyperparathyroidism.

Design: intervention study without control group.

Statistical analysis: we used central tendency and dispersion tests (mean, standard deviation) and the measurement comparison was made by Student's t test.

Patients, materials and methods: over a collection period of two years, we identified subjects with CKD stage 5 on dialysis therapy (hemodialysis or peritoneal dialysis), over 18 years of age, who presented serum iPTH levels greater than $600 \mathrm{pg} / \mathrm{ml}$, and autonomous parathyroid glands hyperplasia (volume greater than $500 \mathrm{~mm}^{3}$ ) confirmed by neck ultrasonography. Under ultrasound guidance ethanol was applied to each hyperplastic gland according to the glandular volume for a maximum of two applications per gland with an interval of 30 days; in case of more than 2 glands compromised, the infiltration of only 2 glands per session was allowed. 15 subjects underwent therapy. In group 1 (G1) were included 5 subjects with one only hyperplastic gland; group 5 (G5) included 10 subjects with more than one hyperplastic gland. Groups 2, 3 and 4 each one with 3, 2 and 5 subjects with two, three and four hyperplastic glands respectively. iPTH levels were assessed at 30, 60, 90 and 120 days after the last application, as well as variables like calcium, phosphorus, calcium-phosphorus product and hemoglobin. Alkaline phosphatase was assessed initially and at 3 months.

Results: intraglandular ethanol administration decreased significantly iPTH values in the total population of subjects from a baseline value of $1263 \pm 554 \mathrm{pg} / \mathrm{ml}$ to $1065 \pm 437 \mathrm{pg} / \mathrm{ml}$ (p value: $0.08), 989 \pm 568 \mathrm{pg} / \mathrm{ml}$ (p value: 0.02$), 1028 \pm 643 \mathrm{pg} / \mathrm{ml}$ (p value: 0.04$)$ and $1139 \pm 749 \mathrm{pg} / \mathrm{ml}(\mathrm{p}$ value: 0.194$)$ to the previously described intervals. In the analysis of groups, we observed that only G1 presented a significant reduction of iPTH values, with a decrease of a mean baseline value of 910 $\pm 508 \mathrm{pg} / \mathrm{ml}$ to $693 \pm 432$ (p value: 0.04 ), $534 \pm 426$ (p: 0.006), $330 \pm 379$ (p: 0.01$)$ and $322 \pm 75$ (p $=.44$ ). In the other groups consolidated in group 5 (G5) with more than 1 hyperplastic gland, iPTH values were not significantly modified, being its initial value $1430 \pm 520 \mathrm{pg} / \mathrm{ml}$ and its subsequent values $1251 \pm 313(\mathrm{p}: 0.287), 1241 \pm 482(\mathrm{p}: 0.255), 1378 \pm 416(\mathrm{p}=0.635)$ and $1489 \pm 591(\mathrm{p}$ : 0.869). The values of calcium, phosphorus, calcium-phosphorus product and alkaline phosphatase decreased in all groups in a not significant way, probably because of the short follow-up time. The most common adverse event was pain and burning sensation that lasted for 5-10 seconds in the injected area, with presence of hypotension in one subject and dysphonia in 3 subjects, resolving with phonoaudiology therapy in the course of three months. All subjects with therapeutic failure were referred to head and neck surgery for parathyroidectomy, finding large fibrous bands during surgery in 8 patients operated to date.

Conclusion: intraglandular ethanol effectively controls secondary hyperparathyroidism in patients with one impaired hyperplastic gland. Subjets with more than one hyperplastic gland should be treated surgically or with an oral calcimimetic, Cinacalcet type. (Acta Med Colomb 2013; 38: 61-67)

Key words: secondary hyperparathyroidism, chronic kidney disease, ethanol.

\section{Introducción}

El hiperparatiroidismo secundario (HPS) es una complicación común en pacientes con enfermedad renal crónica estadio 5 en terapia dialítica (ERC-5-D), y su presencia conlleva a una serie de complicaciones óseas y cardiovasculares que atenta contra la salud de los pacientes (1). Diversos esquemas terapéuticos se han propuesto para el tratamiento del HPS, pero las terapias actuales son incapaces de controlar la síntesis y producción de parathormona en un porcentaje importante de sujetos, inclusive con medicamentos de alto costo que impactan fuertemente en los recursos de la salud destinados para este tipo de pacientes $(2,3)$. La evolución histopatológica del HPS implica la generación inicial de hiperplasia difusa y policlonal de las 
glándulas paratiroides, la cual si no se trata adecuadamente avanza a hiperplasia nodular monoclonal y autónoma $(4,5)$, con reducción muy significativa en el número de receptores al calcio, vitamina $\mathrm{D}(6,7)$ y refractariedad terapéutica. La ecografía de paratiroides principalmente con nuevos ecógrafos de alta resolución permiten identificar fácilmente glándulas paratiroideas hiperplásicas (8), destacándose aquellas con volúmenes mayores a $500 \mathrm{~mm}^{3}$ en las que la hiperplasia nodular autónoma es el patrón predominante, logrando la ecografía sensibilidad del 78,9\% y especificidad del 78,8\% para tal diagnóstico (9-12). Para el grupo de pacientes con hiperplasia nodular el cual es en un alto porcentaje refractario a las terapias clásicas, e incluso a algunas recientes como el cinacalcet $(3,13,14)$, se han planteado diversas terapias antes de llegar a la paratiroidectomía (2). De ellas se han sugerido útiles: aplicación directa percutánea de calcitriol y etanol en glándulas paratiroides hiperplásicas.

La eficacia del etanol intraglandular es controvertida, y se han informado excelentes resultados por algunos autores, pero poca o nula por otros. Los resultados irregulares de respuesta a inyección de etanol percutáneo (IEP) intraglandular pueden ser consecuencia de no haber tenido en cuenta el número de glándulas hiperplásicas que presenten los pacientes, volúmenes glandulares, vascularización ni cifras de PTH intacta.

Con el fin de dar más claridad en este confuso campo, nosotros decidimos evaluar en el servicio de radiología del Hospital Santa Sofía la respuesta a la inyección percutánea de etanol al 99,5\% intraglandular en pacientes con ERC estadio 5 e hiperplasia nodular.

\section{Pacientes, material y métodos}

Durante un periodo de dos años se evaluaron en el servicio de radiología del Hospital Santa Sofía (ManizalesCaldas, Colombia-Sur América) pacientes con ERC-5-D (hemodiálisis o diálisis peritoneal), interconsultados por sospecha de hiperparatiroidismo secundario refractario, mayores de 18 años y quienes presentaran valores de PTH intacta mayores a $600 \mathrm{pg} / \mathrm{mL}$, a pesar de recibir terapia con dieta baja en fosfatos, quelantes de fosfatos y vitamina $\mathrm{D}$ activa (calcitriol). En todos los pacientes se practicó ultrasonografía de cuello, tiroides- paratiroides con equipo de alta resolución (Equipo Phillips Enviisor CHD - Transductor 12 $\mathrm{MHz})$. De 86 pacientes evaluados se detecto en $30(34,88 \%)$ hiperplasia de glándulas paratiroides (Figuras 1 y 2) (8). En 15 el volumen de las glándulas fue mayor a $500 \mathrm{~mm}^{3}$, con lo cual se consideró que cumplían con los criterios recomendados por la sociedad japonesa en el 2006 para selección de pacientes candidatos a aplicación de etanol percutáneo en hiperparatiroidismo secundario (Tablas 1, 2 y 3) (15). A cada uno de ellos por guía ecográfica y previo consentimiento escrito se les aplicó etanol en cada glándula hiperplásica, siendo el volumen aplicado el de la glándula paratiroides intervenida y calculado según la fórmula: axbxcх $\pi / 6$ en $\mathrm{mm}^{3}$, donde $\mathrm{a}, \mathrm{b}$ y c corresponden al diámetro

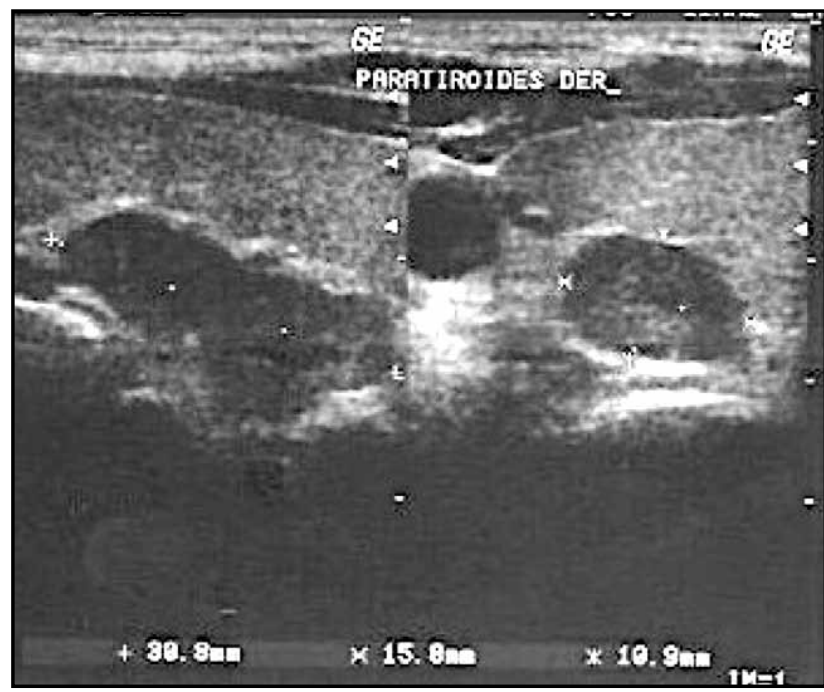

Figura 1. Gran adenoma paratiroideo con volumen de $2536 \mathrm{~mm}^{3}$

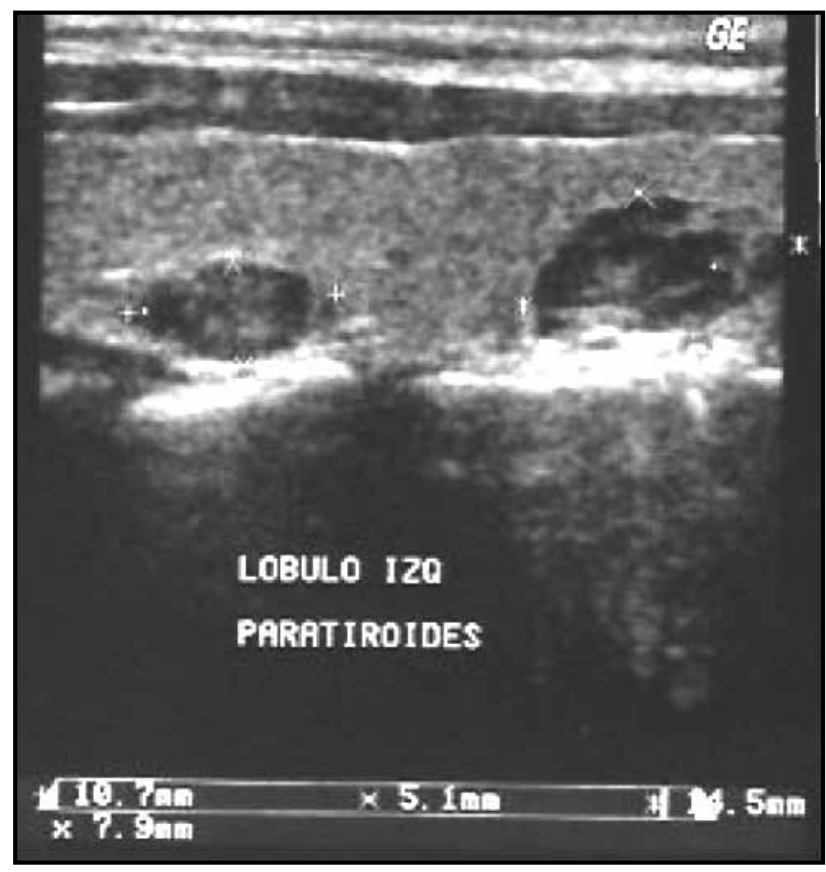

Figura 2. Presencia de dos adenomas izquierdos en un mismo paciente.

Tabla 1. Guía para la aplicación selectiva de terapia con inyección de etanol percutáneo (IEP) para el hiperparatiroidismo secundario (15).

\begin{tabular}{|c|c|}
\hline \multicolumn{1}{|c|}{ Indicaciones para practicar IEP } \\
\hline $1 \quad \begin{array}{l}\text { PTH intacta mayor a 400pg/mL a pesar de tratamiento médico o dificultad en } \\
\text { continuar el tratamiento a causa de hiperfosfatemia e hipercalcemia debido a } \\
\text { tratamiento médico. }\end{array}$ \\
$2 \quad \begin{array}{l}\text { Presencia de glándulas aumentadas de tamaño con sospecha de hiperplasia } \\
\text { nodular confirmada por ecografía. }\end{array}$ \\
\hline $3 \quad$ Consentimiento aceptado para practicar la inyección percutánea de etanol. \\
\hline $\begin{array}{l}\text { IEP está indicada para pacientes quienes llenan tres de las condiciones previas, excepto } \\
\text { para los casos indicados en la Tabla 2. }\end{array}$ \\
\hline
\end{tabular}


Tabla 2. Guía para la aplicación selectiva de terapia con inyección de etanol percutáneo (IEP) para el hiperparatiroidismo secundario (15)

\begin{tabular}{|c|c|}
\hline \multicolumn{2}{|c|}{ Criterios de exclusion para practicar IEP } \\
\hline $1 \quad \begin{array}{l}\text { Presencia de glándulas aumentadas de tamaño en localización donde la punción } \\
\text { bajo guía ultrasonográfica es imposible. }\end{array}$ \\
$2 \quad$ Presencia de parálisis contralateral del nervio recurrente. \\
$3 \quad$ Cirugía previa de cuello requerida para tumor paratiroideo o por otras razones. \\
\hline
\end{tabular}

de las 3 dimensiones de la glándula. Se practicó un máximo de dos aplicaciones por glándula con un intervalo de 30 días (Figura 3). En cada sesión si el número de glándulas fue superior a dos sólo se permitió la infiltración de dos y siempre del mismo lado, continuando a los 30 días con las contralaterales. En el grupo $1(\mathrm{G} 1)$ se incluyeron cinco pacientes con una sola glándula hiperplásica, los grupos 2 (G2), 3 (G3) y 4 (G4) comprendieron tres, dos y cinco pacientes con dos, tres y cuatro glándulas hiperplásicas respectivamente, y se agruparon en el grupo 5 (G5). Los niveles de PTHi se evaluaron a los 30, 60, 90 y 120 días posterior a la ultima aplicación, al igual que las variables calcio, fósforo, producto calcio-fósforo y hemoglobina. La fosfatasa alcalina se evaluó inicialmente y a los tres meses.

La meta fue lograr restaurar los niveles de PTH intacta a valores en rangos considerados satisfactorios (150-300 $\mathrm{pg} / \mathrm{mL})$.

En ningún paciente se practicó gammagrafía con Sestamibi para identificar glándulas ectópicas.

\section{Análisis estadístico}

La información fue procesada en el Programa SPSS versión 15.0 licenciada por la Universidad de Caldas. Para el análisis estadístico se utilizaron pruebas de tendencia central y de dispersión (media, desviación estándar) y con el objetivo de evidenciar diferencias estadísticamente significativas se realizó la comparación de medias a través de la prueba $t$ de Student.

\section{Resultados}

La administración de etanol intraglandular disminuyó los valores de PTH intacta significativamente en la población total de pacientes de un valor inicial de $1263 \pm 554$ a 1065 \pm 437 (p: 0.08), $989 \pm 568$ (p: 0.02), $1028 \pm 643$ (p: 0.04)

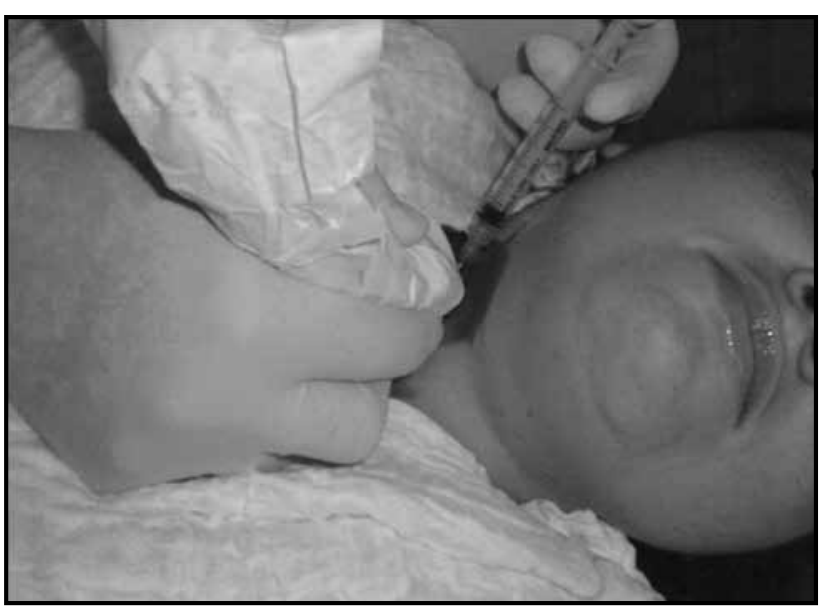

Figura 3. Aplicación percutánea de etanol intraglandular.

y $1139 \pm 749$ (p: 0.194) a los intervalos previamente descritos. En el análisis de grupos se observó que sólo en el G1 se generó reducción significativa en los valores de PTH intacta, con reducción de un valor inicial promedio de 910 \pm 508 pg/mL a $693 \pm 432$ (p: 0.04), $534 \pm 426$ (p: 0.006), $330 \pm 379$ (p: 0.01$)$ y $322 \pm 75$ (p: 0.44). En los otros grupos consolidados en el grupo 5 (G5) con más de una glándula hiperplásica los valores de PTH intacta no se modificaron en forma importante, siendo su valor inicial $1430 \pm 520$, y sus valores posteriores $1251 \pm 313$ (p: 0.287$), 1241 \pm 482$ (p: 0.255$), 1378 \pm 416$ (p: 0.635$)$ y $1489 \pm 591$ (p: 0.869$)$ (Tabla 4). Cuatro pacientes, todos del grupo 1 en algún momento alcanzaron valores de PTH intacta menores a 300 pg/ $\mathrm{mL}$. Los valores de calcio, fósforo, producto calcio-fósforo y fosfatasa alcalina, se disminuyeron en todos los grupos, pero en rangos que no alcanzaron significancia estadística, probablemente por el corto tiempo de seguimiento. El efecto colateral más referido fue dolor y sensación de quemazón en el área infiltrada, que se prolongó por 5-10 segundos, con presencia de hipotensión en una paciente. Disfonía se presentó en tres pacientes, moderada en dos, y severa en uno, resolviéndose con ayuda de fonoaudiología en el curso de tres meses. En ningún paciente se presentó hematoma en el área puncionada. El volumen de las glándulas paratiroides no se modificó significativamente durante el curso del estudio a pesar de las aplicaciones.

Todos los pacientes con fallo terapéutico fueron referidos a cirugía de cuello para que se les practicara paratiroi-

Tabla 3. Características basales de los pacientes intervenidos.

\begin{tabular}{|l|c|c|c|c|c|c|c|}
\hline & Edad (años) & Género & $\begin{array}{c}\text { Tiempo promedio } \\
\text { en diálisis (meses) }\end{array}$ & $\begin{array}{c}\text { Tamaño promedio } \\
\text { nódulos (mm })^{3}\end{array}$ & $\begin{array}{c}\text { PTHi } \\
(\mathbf{p g} / \mathbf{m L})\end{array}$ & $\begin{array}{c}\text { Calcio } \\
(\mathbf{m g} / \mathbf{d L})\end{array}$ \\
\hline Grupo 1 & $61.4 \pm 15.05$ & $\begin{array}{c}\text { M: } 4 \\
\text { H: } 1\end{array}$ & $65.6 \pm 27.80$ & $658 \pm 127.36$ & $910 \pm 508.38$ & $10.39 \pm 0.65$ & $4.39 \pm 0.66$ \\
\hline Grupo 5 & $47.5 \pm 7.70$ & $\begin{array}{c}\text { M: } 5 \\
\text { H:5 }\end{array}$ & $101.4 \pm 42.20$ & $824 \pm 196.23$ & $1430 \pm 520.53$ & $11.18 \pm 0.53$ & $6.06 \pm 1.35$ \\
\hline
\end{tabular}


Tabla 4. Resultados de la aplicación de etanol intraglandular.

\begin{tabular}{|c|c|c|c|c|c|}
\hline Variable PTH Intacta & Inicial en $\mathrm{pg} / \mathrm{mL}$ & 30 días & 60 días & 90 días & 120 días \\
\hline Población total & $1263 \pm 554$ & $1065 \pm 437$ (p:0.08) & $989 \pm 568(\mathrm{p}: 0.02)$ & $1028 \pm 643$ (p:0.04) & $1139 \pm 749(\mathrm{p}: 0.194)$ \\
\hline G1 (1 nódulo) & $910 \pm 508$ & $693 \pm 432(\mathrm{p}: 0.04)$ & $534 \pm 426(\mathrm{p}: 0.006)$ & $330 \pm 379(\mathrm{p}: 0.01)$ & $322 \pm 75(\mathrm{p}: 0.44)$ \\
\hline G2 (2 nódulos) & $1243 \pm 182$ & $1280 \pm 116(\mathrm{p}: 0.68)$ & $1078 \pm 272(\mathrm{p}: 0.32)$ & $1253 \pm 58.3(\mathrm{p}: 0.92)$ & No datos \\
\hline G3 (3 nódulos) & $838 \pm 263$ & $1082 \pm 441(\mathrm{p}: 0.30)$ & $847 \pm 625.8(\mathrm{p}: 0.97)$ & $1161 \pm 459(\mathrm{p}: 0.26)$ & $1075 \pm 837(\mathrm{p}: 0.66)$ \\
\hline G4 (4 nódulos) & $1779.8 \pm 470$ & $1303 \pm 385(\mathrm{p}: 0.13)$ & $1561.5 \pm 416(\mathrm{p}: 0.38)$ & $1538.8 \pm 517(\mathrm{p}: 0.19)$ & $1654 \pm 479(\mathrm{p}: 0.25)$ \\
\hline G5 (+ de 1 nódulo) & $1430 \pm 520$ & $1251 \pm 313(\mathrm{p}: 0.287)$ & $1241 \pm 482(\mathrm{p}: 0.25)$ & $1378 \pm 416(\mathrm{p}: 0.63)$ & $1489 \pm 591(\mathrm{p}: 0.86)$ \\
\hline
\end{tabular}

dectomía quirúrgica, refiriendo los cirujanos la presencia de bandas fibrosas que dificultaron el procedimiento en los hasta ahora ocho pacientes operados.

\section{Discusión}

El tratamiento del HPS en pacientes con ERC abarca una gran variedad de maniobras terapéuticas, las cuales incluyen dieta baja en fosfatos, quelantes de fosfato oral, inhibidores de la absorción intestinal de fosfatos (16), vitamina D activa, agonistas selectivos y no selectivos del receptor de la vitamina $\mathrm{D}$, inhibidores de la remodelación ósea (17), y más recientemente calcimiméticos (cinacalcet).

Sin embargo, en pacientes con ERC-5-D e hiperparatiroidismo secundario, se ha observado que en un porcentaje importante se puede presentar refractariedad terapéutica a las terapias previamente enunciadas, incluyéndose: calcitriol (18-21), agonistas selectivos del receptor de la vitamina D (22-25) y cinacalcet (3,13-15,26-28), ello ocurre principalmente cuando los niveles de PTH intacta son mayores a 500 $\mathrm{pg} / \mathrm{mL}$, y por ultrasonido se detecta presencia de glándulas hiperplásicas en volumen mayor a $500 \mathrm{~mm}^{3}$, lo que obliga a seleccionar otro tipo de terapias.

La introducción del ultrasonido para el estudio de las glándulas paratiroideas cambió radicalmente el enfoque terapéutico del HPS en la ERC. El tamaño normal de las glándulas paratiroides es de sólo $5 \mathrm{~mm}$ x $3 \mathrm{~mm}$ x $1 \mathrm{~mm}$ y contiene un $50 \%$ de grasa $(29,30)$ siendo invisibles a las técnicas actuales de ultrasonido (US), de ahí que cualquier glándula que sea detectada por US se considera anormalmente grande, debiéndose resaltar aquellas con un volumen glandular superior a $500 \mathrm{~mm}^{3}$, ampliamente reconocido por ser compatible con hiperplasia nodular $(9,10-12)$. Estudios han demostrado su superioridad a la tomografía axial computarizada en el diagnóstico de hiperparatiroidismo (30), e igual sensibilidad que la gammagrafía con Sestamibi cuando las glándulas están ubicadas en el cuello (31). Pacientes con valores persistentemente altos de PTH intacta mayores a $600 \mathrm{pg} / \mathrm{mL}$, y con glándulas hiperplásicas detectadas por US con volumen glandular superior a 500 $\mathrm{mm}^{3}(8)$, a la fecha sólo deben recibir como primera opción terapéutica cinacalcet asociado según sus niveles de calcio sérico a calcitriol o agonistas selectivos del receptor de la vitamina D $(15,32)$; en ausencia de respuesta a esta última maniobra se debe considerar otro tipo de terapias entre las cuales resaltan inyección percutánea intraglandular de calcitriol, maxacalcitol, etanol y paratiroidectomía quirúrgica.

Paratiroidectomías quirúrgicas son necesarias en 20,8\% de los pacientes quienes experimentan terapia de hemodiálisis por más de 16-20 años (33). A lo largo del tiempo su práctica ha variado de acuerdo con aparición de medicamentos que puedan impactar en el tratamiento del hiperparatiroidismo, aunque a partir del año 2005 ha resurgido su utilización (34). La paratiroidectomía quirúrgica en manos adecuadas tiene muy baja morbimortalidad $(0,1-1 \%)$ y logra excelentes resultados en cualquiera de sus formas parcial o total con o sin autotrasplante (35-37), siendo notable la rápida mejoría clínica, principalmente en pacientes con altos niveles de fosfatasa alcalina (38).

El etanol intraglandular para el tratamiento del hiperparatiroidismo secundario fue utilizado por primera vez en 1985 por Solbiati y cols como una alternativa a la paratiroidectomía en 12 pacientes urémicos de alto riesgo quirúrgico o que se rehusaban a que se les practicara, reportándose efectos clínicos y bioquímicos satisfactorios en la mayoría de los pacientes $(39,40)$, iguales resultados reportan Kitaoka y cols en Japón quienes intervinieron nueve pacientes con glándulas paratiroides de gran tamaño (41), Kakuta y cols en 46 pacientes seguidos durante un año con excelente respuesta en el $80 \%$ de ellos $(42,43)$ y Tanaka y cols en 33 pacientes (44).

Pero en otros estudios se han reportado resultados muy diferentes; Fletcher y cols en el Reino Unido informan sólo beneficio de la inyección de etanol percutánea (IEP) en 50\% de los 22 pacientes intervenidos (45), Nakamura y cols en $56 \%$ de 74 pacientes (46), Cintin y cols en $60 \%$ de nueve pacientes (47), y ausencia completa de respuesta al IEP en el estudio de Barros Gueiros y cols (48).

Los resultados irregulares de respuesta a etanol intraglandular pueden ser consecuencia de no haber tenido en cuenta el número de glándulas hiperplásicas que presentaban los pacientes, volumen glandular, vascularización, ni cifras de PTH intacta.

Tanaka y cols en 28 pacientes, 21 con una a dos glándulas hiperplásicas lograron excelentes resultados en $81,2 \%$ de los casos, mientras sólo en $20 \%$ de pacientes con tres a cuatro glándulas hiperplásicas (49), Fassi y cols en Argentina 
observaron que en 25 pacientes tratados con una sola glándula hiperplásica lograron excelente respuesta, a diferencia de los que tenían más de una glándula hiperplásica en los que la respuesta fue en sólo $30.8 \%$ (50), Onoda y cols en 30 pacientes evaluados, en aquellos con una a dos glándulas se logró respuesta en $68.4 \%$ vrs sólo en $36.4 \%$ en tres a cuatro glándulas (51), Koiwa y cols en 321 pacientes tratados con IEP concluyeron que solo pacientes con una glándula hiperplásica lograban buena respuesta, la cual perduraba durante un periodo mayor de tiempo (52).

En Colombia el grupo de De Nubila y cols en el cual está incluido el nefrólogo Jaime Mercado, en el año 2010 reportaron su experiencia con IEP en 15 pacientes con ERC, hiperparatiroidismo secundario y adenomas, sin lograr respuesta terapéutica en $40 \%$ de los pacientes, y en $60 \%$ restante alguna respuesta, que fue exitosa en $33.3 \%$ de ellos, subóptima en $13.3 \%$ y no satisfactoria en $13.3 \%$. Al analizar los pacientes por número de adenomas intervenidos este grupo detecto que sólo aquellos pacientes con menos de 1.8 lograron exitosa respuesta terapéutica (53). También en Colombia Romero y cols presentan un reporte de caso, en el cual una paciente de 84 años con ERC e hipercalcemia, y con un adenoma paratiroideo asociado a múltiples comorbilidades que contraindicaban manejo quirúrgico, es intervenida con IEP en tres oportunidades lográndose normalización en los valores de PTH intacta (54).

En nuestro estudio se encontró una excelente respuesta terapéutica sólo en pacientes con una glándula hiperplásica, estos resultados son el producto de haber considerado el número de glándulas paratiroides afectadas y su volumen, hallazgos semejantes a lo reportado en diversos trabajos internacionales. Nuestro estudio permite dar mayor claridad a este controvertido aspecto del manejo del hiperparatiroidismo secundario con IEP. La terapia es muy bien tolerada y los efectos colaterales que genera son fácilmente manejables.

\section{Conclusión}

El etanol intraglandular controla efectivamente el hiperparatiroidismo secundario refractario de pacientes con ERC estadio 5 en terapia dialítica, pero sólo en pacientes con una glándula hiperplásica. Pacientes con más de una glándula hiperplásica deben ser referidos precozmente a cirugía de cuello para tratamiento quirúrgico, o darles previamente la oportunidad de recibir un calcimimético tipo Cinacalcet.

\section{Referencias}

1. Block GA, Klassen PS, Lazarus JM, Ofsthun N, Lowrie EG, Chertow GM. Mineral metabolism, mortality and morbidity in maintenance hemodialysis patients. J Am SocNephrol 2004; 15: 2208-2218.

2. Komaba H, Shiizaki K, Fukagawa M. Pharmacotherapy and interventional treatments for secondary hyperparathyroidism: current therapy and future challenges. Expert Opin Biol Ther 2010; 10: 1729-1742.

3. Okada M, Tominaga Y, Izumi K, Nobata H, Yamamoto T, Hiramitsu T, Tsujita M, Goto N, Nanmoku K, Watarai T, Uchida K. Tertiary hyperparathyroidism resistant to Cinacalcet treatment. Ther Apher Dial 2011; 15 (Suppl 1): 33-37.

4. Tominaga $\mathbf{Y}$, Kohara S, Namii $\mathbf{Y}$ et al. Clonal analysis of nodular parathyroid hyperplasia in renal hyperparathyroidism. World J Surg 1993; 20: 744-50.

5. Tominaga Y, Tsuzuki T, Uchida $\mathbf{K}$ et al. Expression of PRAD1/cyclinD1, retinoblastoma gene products, and Ki67 in parathyroid hyperplasia caused by chronic renal failure versus primary adenoma. Kidney Int 1999; 55: 1375-83.

6. Fukuda N, Tanaka H, Tominaga Y, Fukagawa M, Kurokawa K, Seiko Y. Decreased 1,25-dihydroxyvitamin D3 receptor density is associated with a more severe form of parathyroid hyperplasia in chronic uremic patients. J Clin Invest 1993; 92: 1436-43

7. Gogusev J, Duchambon P, Hory B et al. Depressed expression of calcium receptor in parathyroid gland tissue of patients with hyperparathyroidism. Kidney Int 1997; 51: 328-36.

8. Restrepo C A, Santacruz D, Castillo C E, Chacon J A. Detección de hiperplasia de paratiroides por ultrasonografía y correlación con signos clínicos y de laboratorio en pacientes con enfermedad renal crónica. Rev Colomb Radiol 2011; 22: 3341-3347

9. Tominaga Y, Tanaka Y, Sato K et al. Histopathology, pathophysiology and indications for surgical treatment of renal hyperparathyroidism. Semin Surg Oncol 1997; 13: 78-86.

10. Kakuta T, Tanaka R, Kanai G, Miyamoto Y, Inagaki M, Suzuki H, Fukugawa M, Saito A. Relationship between the weight of parathyroid glands and their secretion of parathyroid hormone in hemodialysis patients with secondary hyperparathyroidism. Ther Apher Dial 2008; 12: 385-390.

11. Tominaga Y, Matsuoka S, Sato T, Uno N, Goto N, Katayama A, Haba T, Uchida K. Clinical features and hyperplastic patterns of parathyroid glands in hemodialysis patients with advanced secondary hyperparathyroidism refractory to maxacalcitol treatment and required parathyroidectomy. Ther Apher Dial 2007; 11: 266-273.

12. Matsuoka S, Tominaga Y, Sato T, Uno N, Hiramitu T, Goyo N, Nagasaka T, Uchida K. Relationship between the dimension of parathyroid glands estimated by ultrasonography and the hyperplastic pattern in patients with renal hyperparathyroidism. Ther Apher Dial 2008; 12: 391-395.

13. Yamamoto M, Ogata H, Mizobuchi M, Yoshida N, Kumata-Maeta C, Koiwa F, Shishido K, Kinugasa E. Number of enlarged parathyroid glands might be a predictor of cinacalcet response in advanced secondary hyperparathyroidism. Clin Exp Nephrol 2012; 16: 292-299.

14. Tanaka M, Nakanishi $S$, Komaba $H$, Itho $K$, Matsushita $K$, Fukugawa $M$ Association between long term efficacy of cinacalcet and parathyroid gland volume in haemodialysis patients with secondary hyperparathyroidism. NDT Plus 2008; 1 (suppl 3): iii49-iii53.

15. Kitaoka M. Changes in interventional treatment-from the viewpoint of percutaneous Ethanol injection therapy. Ther Apher Dial 2008; 12 (suppl 1): S16-S20.

16. Restrepo C A, Cruz J. Efectividad y seguridad del acido nicotínico en el tratamiento de la hiperlipidemia asociada a hiperfosfatemia en pacientes con enfermedad renal crónica. Nefrologia 2008; 28: 61-66.

17. Restrepo C A, Manjarres G. Controversia en relación con el uso de bifosfonatos en pacientes con enfermedad renal crónica. Acta Med Colomb 2009; 34: 176-184.

18. Tominaga Y, Numano M, Uchida K, Katayama A, Haba T, Asano H, Sato K, Tanaka Y, Takagi H. Parathyroidectomy for patientswith renal hyperparathyroidism refractoryto calcitriol pulse therapy. J Bone Miner Met 1994; 12 Suppl. 1: S99-S104.

19. Katoh N, Nakayama M, Shigematsu T, Yamamoto H, Sano K, Saito I, Nakano H, Kasai K, Kubo H, Sakai S, Kawaguchi Y, Hosoya T. Presence of sonographically detectable parathyroid glands can predict resistance to oral pulsed dose calcitriol treatment of secondary yhyperparathyroidism. Am J Kidney Dis 2000; 35: 465-468

20. Fukagawa M, Kitaoka M, Yi H, Fukuda N, Matsumoto T, Ogata E, Kurokawa. Serial evaluation of parathyroidsizeby ultrasonography is another useful marker for the longterm prognosis of calcitriol pulse theraphy in chronic dyalisis patients. Nephron 1994; 68: 221-228.

21. Quarles L D, Yohay D A, Carroll B A, Spritzer C E, Minda S A, BartholomayD, Lobaugh B A. Prospective trial of pulse oral versus intravenous calcitriol treatment of hyperparathyroidism in ESRD. Kidney Int 1994; 45: 1710-1721.

22. Tominaga $Y$, Matsuoka $S$, Sato $T$, Uno $N$, Goto $N$, Katayama A, Haba $T$, Uchida K. Clinical features and hyperplastic patterns of parathyroid glands in hemodialysis patients with advances secondary hyperparathyroidism refractory to maxacalcitol treatment and required parathyroidectomy. Ther Apher Dial 2007; 11: 266-273

23. Tominaga Y, Inaguma D, Matsuoka S, Tahara H, Kukita K, Kurihara S, Onoda N, Tsuruta Y, Tsutsui S, Ohta K, Kuwahara M, Tanaka M, Nishizawa Y. Is the volumen of the parathyroid gland a predictor of maxacalcitol response in advanced secondary hyperparathyroidism. Ther Apher Dial 2006; 10: 198-204.

24. Okuno S, Ishimura E, Kitatani K, Chou H, Nagasue K, Mackawa S, Izumotani T, Yamakawa T, Imanishi Y, Shoji T, Inaba M, Nishizawa. Relationship between parathyroid gland size and responsiveness to maxacalcitol theraphy in patients with secondary hyperparathyroidism. Nephrol Dial Transplant 2003; 18: $2613-2621$ 
25. Vulpio C, Maresca G, Distasio E, Cacaci S, Panocchia N, Luciani G, Bossola M. Switch from calcitriol to paricalcitol in secondary hyperparathyroidism of hemodialysis patients: responsiveness es related to parathyroid gland size. Hemodialysis Int 2011; 15: 69-78.

26. Hirai T, Nakashima A, Takasugi N, Yorioka N. Association of nodular hyperplasia with resistance to cinacalcet therapy for secondary hyperparathyroidism in hemodialysis patients. Ther Apher Dial 2012; 14: 577-582.

27. Vulpio C, Bossola M, De Gaetano A, Maresca G, Di Stasio E, Zagaria L, Luciana G, Giordano A, Castagneto M. Parathyroid gland ultrasound patterns and biochemical finding after one year cinacalcet treatment for advanced secondary hyperparathyroidism. Ther Apher Dial 2009; 14: 178-185.

28. Hirai T, Nakashima A, Takasugi N, Yorioka N. Response of secondary hyperparathyroidism to cinacalcet depends on parathyroid size. Nephron Clin Pract 2010; 114: c187-c193.

29. Wang C. The anatomic basis of parathyroid surgery. Ann Surg 1976; 183: 271-275.

30. Takebayashi S, Matsui K, Onohara Y, Hidai H. Sonography for early diagnosis of enlarged parathyroid glands in patients with secondary hyperparathyroidism. AJR 1987; 148: 911-914.

31. Haber R S, Kim C K, Inabnet $\mathbf{W} \mathbf{B}$. Ultrasonography for preoperative localization of enlarged parathyroid glands in primary hiperparathyroidism: comparison with 99mtechnetium sestamibi scintigraphy. Clin Endocrinology 2002; 57: 241-249

32. Restrepo C A, Buitrago C, Torres J, Serna J. Desórdenes óseos y minerales en la enfermedad renal crónica. Nefrología Básica 2. Editorial la Patria 2012, pag. 249-256, Manizales.

33. Malberti F, Marcelli D, Conte F, Limido A, Spotti D, Locatelli F. Parathyroidectomy in patients on renal replacement therapy: An epidemiologic study. J Am Soc Nephrol 2001; 12: 1242-1248.

34. Li S, Chen Y-W, Peng Y, Foley R N, St. Peter W L. Trends in parathyroidectomy rates in US hemodialysis patients from 1992 to 2007. Am J Kidney Dis 2011; 57: 602-611.

35. Rivero V P, Pison E, Tamayo J M P, Claver F C, Camacho M G, Huelva A B. Cirugia del hiperparatiroidismo secundario y terciario: 11 años de experiencia. Acta Otorrinolaringol Esp 2002; 53: 418-422

36. Tominaga Y, Numano M, Tanaka Y, Uchida K, Takagi H. Surgical treatment of renal hyperparathyroidism. Semin Surg Oncol 1997; 13: 87-96.

37. De Francisco Angel L M, Fresnedo G F, Rodrigo E, Piñeira C, Amado J A, Arias M. Parathyroidectomy in dialysis patients. Kidney Int 2002; 61 (suppl 80): S161-S166.

38. Neonakis E, Wheeler M H, Krishnan H, Coles G A, Davies F, Woodhead J S. Results of surgical treatment of renal hyperparathyroidism. Arch Surg 1995; 130: 643-648.

39. Solbiati L, Giangrande A, De Prada L, Belloti E, Cantu P, Ravetto C. Percutaneous ethanol injection of parathyroid tumors under US guidance: treatment for secondary hyperparathyroidism. Radiology 1985; 155: 607-610.

40. Giangrande A, Cantu P, Solbiati L, Ravetto C. Ultrasonically guided fineneedle alcohol injection as an adjunctto medical treatment in secondary hyperparathyroidism. Proc Eur Dial Transplant Eur Ren Assoc 1985; 21: 895-901.
41. Kitaoka M, Fukagawa M, Ogata E, Kurokawa $K$. Reduction of functioning parathyroid cell mass by ethanol injection in chronic dialysis patients. Kidney Int 1994; 46: 1110-1117.

42. Kakuta T, Fukagawa M, Fujisaki T, Hida M, Suzuki H, Sakai H, Kurokawa K, Saito A. Prognosis of parathyroid function after successful percutaneous ethanol injection therapy guided by color Doppler flow mapping in chronic dialysis patients. Am J Kidney Dis 1999; 33: 1091-1099,

43. Kakuta T, Kunimatsu K, Tadaki F, Noguchi M,Abe Y, Sakai H, KurokawaK, Saito A. Long term prognosis of parathyroid function after successful percutaneous ethanol injection therapy (PEIT) guided by color Doppler flow mapping in chronic dialysis patients. Biomed Pharmacother 2000; 54 (suppl 1): 60s-65s.

44. Tanaka R, KakutaT, Fujisaki T, Tanaka S, Sakai H, Kurokawa K, Saito A. Long term (3 years) prognosis of parathiroid function on chronic dialysis patients after percutaneous ethanol injection therapy guided by colour Doppler ultrasonography. Nephrol Dial Transplant 2003; 18 (suppl 3): iii58-61.

45. Fletcher S, Kanagasundaram N S, Rayner H C, Irving H C, Fowler R C, Brownjohn A M, Turney J H, Will E J, Davison A M. Assessment of ultrasound guided percutaneous ethanol injection and parathyroidectomy in patients with tertiary hyperparathyroidism. Nephrol Dial Transplant 1998; 13: 3111-3117.

46. Nakamura M, Fuchinoue $S$, Teraoka S. Clinical experience with percutaneous ethanol injection therapy in hemodialysis patients with renal hyperparathyroidism. Am J Kidney Dis 2003; 42: 739-745.

47. Cintin C, Karstrup S, Ladefoged S D, Joffe P. Tertiary hyperparathyroidism treated by percutaneous fine needle ethanol injection. Nephron 1994; 68: 217-220.

48. De Barros Gueiros J E, Chammas M C, Gerhard R, Días Boilesen C F, De Olivera I R, AffonsoMoyses R M, Jorgetti V. Percutaneous ethanol and calcitriol injection therapy are ineffective in treating severe secondary hyperparathyroidism. NDT 2004; 19: 657-663.

49. Tanaka M, Itoh K, Matsushita K, Matsushita K, Fukagawa M. Efficacy of percutaneous ethanol injection therapy for secondary hyperparathyroidism in patients on hemodialysis as evaluated by parathyroid hormone levels according to K/DOQI guidelines. Ther Apher Dial 2005; 9: 48-52.

50. Fassi J, Lambertini R, Farias P, Blejman O, Rosa Diez G, Algranati S Plantalech L. Treatment of uremic hyperparathyroidism with percutaneous ethanol injection. Nephron Clin Pract 2005; 101: c53-57.

51. Onoda N, Kashiwagi T, Nakamura T, Niitsu Y, Omata M, Kurihara S Parathyroid interventions for secondary hyperparathyroidism in hemodialyzed patients. Ther Apher Dial 2005; 9 (suppl 1): S11-S15.

52. Koiwa F, Kakuta T, Tanaka R, Yumita S. Efficacy of percutaneous ethano injection therapy (PEIT) is related to the number of parathyroid glands in haemodialysis patients with secondary hyperparathyroidism. Nephrol Dial Transplant 2006; 22: 522-528.

53. De Nubila E, Vega J, Garcia L, Mercado J. Efectividad terapéutica y seguridad de la ablación de adenomas paratiroideos con inyección percutánea de etanol bajo guía ecográfica en pacientes con hiperparatiroidismo secundario refractario a tratamiento médico con insuficiencia renal crónica. Rev Colomb Radiol 2010 21: 2945-2956.

54. Romero J, Barragan C, Chica G. Alternativa no quirúrgica para el tratamiento del adenoma de glándula paratiroides. Ablación percutánea con alcohol guiada con ultrasonido. Acta Med Colomb 2011; 36: 41-44. 